\title{
Mudanças no self de carreira em estudantes universitários ${ }^{1}$
}

\author{
Ana Daniela Silva \\ Maria do Céu Taveira ${ }^{2}$ \\ Eugénia Ribeiro \\ Universidade do Minho, Braga, Portugal
}

\begin{abstract}
Resumo: Apresenta-se um estudo que tem como objectivo avaliar as mudanças no self de carreira de estudantes universitários do início para o final do último ano de graduação. Para tal recorreu-se a um estudo de medidas repetidas dos índices de uma Grelha de Repertório da Carreira (GRC), desenvolvido para este propósito. Esta grelha avalia a relação estabelecida pelos estudantes entre construtos vocacionais, aspectos do self e figuras determinantes da construção da sua identidade. Do estudo participaram 80 estudantes de graduação da Universidade do Minho, no noroeste de Portugal, inscritos pela primeira vez no último ano do curso de licenciatura, dos quais 49 são mulheres $(61,25 \%)$ e 31 são homens $(38,75 \%)$, com idades compreendidas entre os 21 e os 45 anos $(M=23,9, D P=4,31)$. Os resultados evidenciam variações intraindividuais, na flexibilidade cognitiva e definição do self de carreira, inferiores no segundo momento de avaliação.
\end{abstract}

Palavras-chave: identidade, ensino superior, estudantes universitários, desenvolvimento profissional.

\section{Changes in the career self of college students}

\begin{abstract}
This study aimed to assess changes in the career self of college students, from the beginning to the end of the last year of the program. For that, a study of repeated measures of the indices of a Career Repertory Grid (GRC), specially developed for this purpose, was conducted. This grid assesses the relationships established by students between vocational constructs, aspects of the self and determinant people in the construction of their identity. A total of 80 undergraduate students from the University of Minho, Northwest of Portugal, who where registered for the first time in the last year of the program participated in the study. Of these, 49 were women (61.25\%) and 31 were men (38.75\%) with ages ranging from 21 to 45 years old $(M=23.9 ; S D=4.31)$. Results evidence intra-individual changes in cognitive flexibility and in the definition of the career self, which decreased in the second assessment.
\end{abstract}

Keywords: identity, higher education, college students, professional development.

\section{Cambios del self de carrera en estudiantes universitarios}

Resumen: Estudio que tuvo como objetivo evaluar los cambios del self de carrera profesional de los estudiantes del comienzo al final del último año de diplomatura. Para tal, se llevó a cabo un estudio de mediciones repetidas de los índices de una Rejilla de la Carrera (GRC) desarrollada para el propósito. Esta Rejilla evalúa la relación establecida por los estudiantes entre constructos vocacionales, dimensiones del self y de relación con los otros envueltos en la construcción de su identidad. En el estudio participaron 80 estudiantes de postgrado de la Universidad de Miño, en el noroeste de Portugal, inscripto por primera vez en el último año del curso de licenciatura de los cuales 49 son mujeres $(61,25 \%)$ y 31 son hombres $(38,75 \%)$, de 21 a 45 años de edad $(M=23,9, S D=4,31)$. Los resultados muestran variaciones intra individuales en la flexibilidad cognitiva y la definición del self de carrera, inferiores en el segundo momento de la evaluación.

Palabras clave: identidad, educación superior, estudiantes universitarios, desarrollo profesional.

O ensino superior deve ser considerado como um período transitório da vida dos estudantes. No entanto, todos nós sabemos quão importante pode ser esta transição na vida de uma pessoa, quer a nível académico, quer a nível social, quer mesmo a nível emocional e pessoal. É um contexto que pode e deve facilitar e promover o desenvolvimento dos indivíduos em várias dimensões da sua existência e confrontar os estudantes, mulheres e homens, com diferentes ideias, experiências, modelos e papéis de vida, preparando a entrada progressiva na vida adulta. Neste contexto, embora o projecto existencial dos jovens adultos estudantes do ensino superior integre diversas dimensões, a construção da identidade

1 Este texto foi revisado seguindo Acordo Ortográfico da Língua Portuguesa (1990), em vigor a partir de $1^{\circ}$ de janeiro de 2009.

2 Endereço para correspondência:

Profa. Dra. Maria do Céu Taveira. Universidade do Minho. Escola de Psicologia. Campus de Gualtar. 4710-057. Braga. Portugal. E-mail: ceuta@iep.uminho.pt vocacional ocupa um lugar preponderante. Este processo envolve o desenvolvimento de tarefas vocacionais, designadamente a de inserção pré-profissional e profissional, e a concretização de projectos mais abrangentes que se desenvolvem ao longo de todas as restantes etapas da vida (Leitão, Paixão, Silva, \& Miguel, 2000).

De acordo com a teoria do construtivismo pessoal, a identidade pessoal (ou self) é definida como um sistema de dimensões de significado pessoal, por meio das quais a pessoa dá sentido aos acontecimentos que antecipa e vive no fluxo da sua existência. Este sistema desenvolve-se, no contexto das relações sociais significativas, e modifica-se face à oportunidade do confronto com experiências de novidade pessoal e interpessoal. Como o mundo em que vivemos não é estático, o processo de viver convida-nos, frequentemente, a rever o sistema de dimensões a partir do qual significamos a nossa existência e desafia-nos à transformação do nosso self. No entanto, para que este processo de transformação ocorra de um modo funcional, é essencial que o sistema de 
significação, ou seja o self, mantenha um sentido de continuidade e coerência, geralmente garantido por dimensões de significado mais centrais (Fernandes, Senra, \& Feixas, 2009). As perspectivas construtivistas mais recentes, sobre o desenvolvimento da identidade, tendem a sublinhar o carácter transformacional do self (Brott, 2001; Lent, Brown, \& Hackett, 2002). Este é considerado como um projecto que se actualiza no contacto com os outros e com os contextos de vida, mediado, todavia, pelas limitações que as organizações cognitivas mais nucleares impõem à pessoa (Fernandes \& Gonçalves, 1997). Concretamente, e de acordo com o paradigma do construtivismo pessoal (Kelly, 1955), a forma como as pessoas percebem o mundo joga um papel importante nas decisões que estas tomam e nos comportamentos que assumem. Paralelamente, vários autores do desenvolvimento humano defendem que este se processa ao longo de um contínuo, hierarquicamente organizado, progredindo em direcção a níveis de maior diferenciação e integração ou complexidade (Harter, 1985, 1988, 1999, 2003; Kohlberg, 1990; Sanford, 1962). O ensino superior parece constituir um contexto propício a estes processos. Com efeito, autores, como Marcia (1966, 1976,1980), procuraram demonstrar que a transição pelo ensino superior é, por excelência, um período propício ao desenvolvimento pessoal, em que os estudantes exploram, de forma mais ampla e variada, as questões associadas à construção e definição da sua identidade e assumem compromissos mais firmes e definitivos nas áreas vocacional, ideológica (religiosa e política) e interpessoal/ sexual das suas vidas. A este respeito, pesquisas recentes com estudantes universitários apontam para um desconhecimento acerca das profissões (Silva, 2008), bem como pouca identidade com a área de trabalho (Bardagi, 2007).

Nesse sentido, torna-se relevante o desenvolvimento de metodologias de avaliação do self de carreira desses estudantes.

O self de carreira é definido por Savickas (2004) como subconjunto organizado do universo cognitivo de uma pessoa, permitindo-lhe identificar e discriminar os papéis de trabalho que constituem uma experiência fulcral. Por isso, esta estrutura permite às pessoas realizarem escolhas coerentes entre alternativas comportamentais. É construído com base na autorreflexão que a linguagem torna possível e é responsável pelo carácter subjectivo que o indivíduo confere à carreira. $\mathrm{Ou}$ seja, permite aos indivíduos produzirem um significado e utilizarem este processo de produção de significados para orientar o seu próprio comportamento, de uma forma proactiva e não apenas como uma mera reaç̧ão a estímulos ambientais.

Nesse contexto, este estudo pretende avaliar as mudanças no self de carreira de estudantes universitários, do início para o final do último ano de licenciatura. Para tal, recorreu-se a um estudo de medidas repetidas dos índices de uma grelha de repertório (GRC) desenvolvida para o efeito (Silva \& Taveira, 2005; Silva, 2008). A grelha de repertório é uma metodologia construtivista derivada do Role Construct Repertory Test construído por Kelly (1955), com o objectivo de explorar a construção de significados pessoais, ou seja, a estrutura e o conteúdo das teorias implícitas ou redes de significado, por meio das quais percebemos e actuamos no quotidiano. Consiste numa entrevista estruturada e orientada para a identificação das dimensões de significado que o sujeito usa para dar sentido ao seu mundo e a partir das quais se diferencia dos outros significativos (Fernandes, 2001; Winter, 2003). A informação recolhida, durante a entrevista, é posteriormente organizada e formalizada numa matriz que inclui as dimensões de significado identificadas (em linhas horizontais), os elementos ou outros significativos sobre os quais recai a construção de significado (em linhas verticais) e um sistema de pontuação, por meio do qual o participante do estudo pontua cada elemento relativamente a cada significado. Dada a formalização da informação numa matriz deste tipo, esta metodologia assume o nome de grelha de repertório. A grelha utilizada neste estudo pretende compreender a estrutura do sistema de construção num âmbito específico da experiência humana - a carreira. Desta forma, a análise matemática das associações entre os elementos e construtos da grelha permite obter índices que explicam como os estudantes organizam significados acerca das suas carreiras. Estes índices calculados com base na análise de correspondências serão explicados detalhadamente, mais à frente, neste artigo. Perceber mudanças nesta forma de organizar a informação acerca da carreira, ao longo do último ano de licenciatura, permite-nos antecipar possíveis formas de os estudantes lidarem com este momento significativo das suas vidas, especialmente, no que diz respeito às exigências da integração socioprofissional que se antecipa com o finalizar de um curso.

\section{Método}

\section{Participantes}

A amostra é composta por 80 estudantes de graduação da Universidade do Minho, no noroeste de Portugal, inscritos pela primeira vez no último ano do curso de licenciatura, no ano lectivo de 2005/2006. Dos 80 estudantes, 49 são mulheres $(61,25 \%)$ e 31 são homens $(38,75 \%)$, com idades compreendidas entre os 21 e os 45 anos, sendo a média de 23,9 anos, e o desvio-padrão de 4,31. Estes alunos provêm de 27 cursos diferentes, organizados no presente estudo, nas áreas de estudo sugeridas pela Classificação Nacional de Áreas de Educação e Formação publicadas no Diário da República, Portaria $n^{\circ}$ 256/2005 de 16 de março: Ciências Sociais Comércio e Direito (46\%), Educação (17\%), Artes e Humanidades (14\%), Ciências, Matemática e Informática (14\%) e Engenharia, Indústrias Transformadoras e Construção (9\%).

\section{Instrumentos}

Trata-se da Grelha de Repertório da Carreira (GRC) (Silva \& Taveira, 2005), que consiste numa entrevista estruturada e formalizada numa original grelha $16 \times 15$, em que os participantes na investigação pontuam um conjunto de 
Silva, A. D., Taveira, M. C., \& Ribeiro, E. (2009). Mudanças no self de carreira.

elementos interpessoais elicitados pelo investigador (por exemplo, mãe, pai, irmão, professor), face a um conjunto de construtos bipolares fornecidos pelo investigador (por exemplo, Dependente dos outros versus Autónomo; Maior tendência para alcançar objectivos versus Menor tendência para alcançar objectivos), usando uma escala de 7 pontos, sendo o valor 4 um ponto médio (por exemplo, 1-Muito dependente dos outros e 7-Muito Autónomo). Daqui resulta uma matriz de cotações para cada participante, com as colunas a representarem pessoas significativas do mundo interpessoal, e as linhas a representarem construtos ou dimensões de significado em torno da carreira (Figura 1). Os elementos interpessoais são elicitados e os construtos são fornecidos pelo investigador. Os 15 construtos fornecidos resultaram da revisão da literatura do desenvolvimento e escolha de carreira.

\begin{tabular}{|c|c|c|c|c|c|c|c|c|c|c|c|c|c|c|c|}
\hline Eu Ideal & $\forall$ & - & - & $r$ & $\forall$ & $m$ & - & - & - & - & - & - & - & - & $\forall$ \\
\hline Eu em 10 anos & + & $\sim$ & - & 6 & 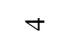 & $m$ & - & - & - & - & - & - & - & - & in \\
\hline Eu em 5 anos & + & $\sim$ & - & 6 & 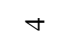 & $m$ & - & - & - & - & - & - & - & - & in \\
\hline Eu em 6 meses & + & - & - & $r$ & $\forall$ & $m$ & - & - & - & - & - & - & - & - & $n$ \\
\hline $\begin{array}{c}\text { Eu antes de entrar na } \\
\text { UM }\end{array}$ & $r$ & $\sigma$ & $\nabla$ & - & $\sigma$ & $\sigma$ & - & $\nabla$ & $\nabla$ & $\nabla$ & $\nabla$ & $m$ & N & $\nabla$ & $\nabla$ \\
\hline $\begin{array}{l}\text { Homem que desag. em } \\
\text { termos de carreira }\end{array}$ & $\nabla$ & $\sigma$ & $\nabla$ & $n$ & $\sigma$ & $\sigma$ & $n$ & $r$ & $r$ & $n$ & $r$ & $\nabla$ & $\nabla$ & 0 & $\nabla$ \\
\hline $\begin{array}{l}\text { Mulher que desag. em } \\
\text { termos de carreira }\end{array}$ & $\nabla$ & $\nabla$ & $\nabla$ & $\nabla$ & $\sigma$ & $\sigma$ & $n$ & $r$ & 6 & $m$ & 6 & $\nabla$ & $\nabla$ & $n$ & $\nabla$ \\
\hline $\begin{array}{l}\text { Homem que agrada } \\
\text { em termos de carreira }\end{array}$ & $\nabla$ & $m$ & $\nabla$ & $\nabla$ & - & † & - & - & - & - & - & - & - & - & $\nabla$ \\
\hline $\begin{array}{l}\text { Mulher que agrada em } \\
\text { termos de carreira }\end{array}$ & - & - & $\nabla$ & $\nabla$ & - & + & - & - & - & - & - & - & - & - & $\nabla$ \\
\hline Professor & - & - & $\theta$ & $\nabla$ & - & 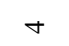 & - & - & - & - & - & - & - & - & 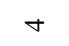 \\
\hline Companheiro & t & - & - & 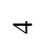 & t & - & - & - & - & - & - & - & - & - & + \\
\hline Pessoa Significativa & N & - & N & 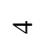 & - & 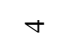 & - & - & - & - & - & - & - & - & 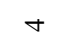 \\
\hline Irmão/Irmã & $\theta$ & $n$ & - & $\checkmark$ & 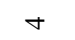 & 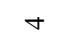 & - & $\checkmark$ & $m$ & $\forall$ & $\nabla$ & 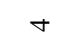 & $m$ & $m$ & $\nabla$ \\
\hline Pai & $r$ & $r$ & - & $\nabla$ & t & $r$ & - & $n$ & $\sim$ & $\nabla$ & + & $\forall$ & + & $\forall$ & $n$ \\
\hline Mãe & $r$ & $r$ & - & $\nabla$ & t & - & - & $n$ & $\sim$ & 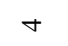 & + & $\forall$ & 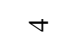 & $\forall$ & - \\
\hline \multirow[t]{3}{*}{ Eu Actual } & + & - & - & $\nabla$ & t & $m$ & - & - & $\sim$ & $\sim$ & $\sim$ & - & - & $\sim$ & $m$ \\
\hline & 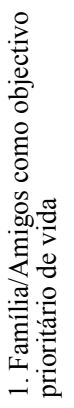 & 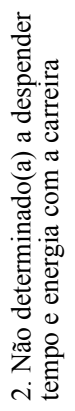 & 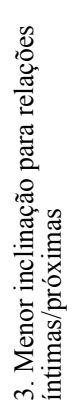 & 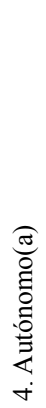 & 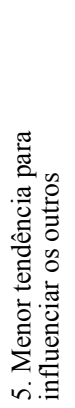 & 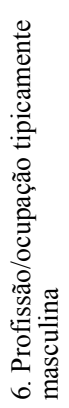 & 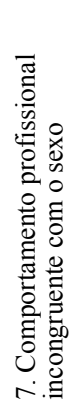 & 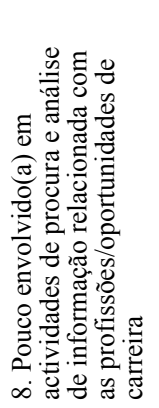 & 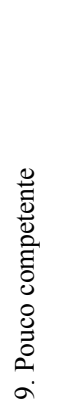 & 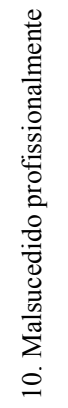 & 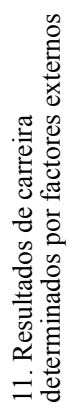 & 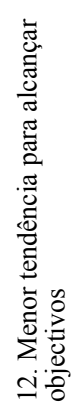 & 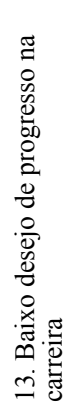 & 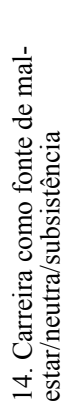 & 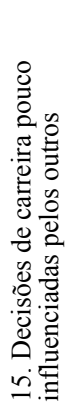 \\
\hline & 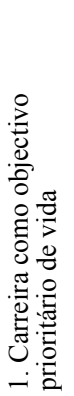 & 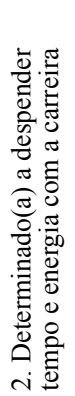 & 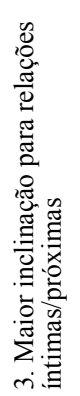 & $\begin{array}{l}0 \\
0 \\
0 \\
0 \\
0 \\
0 \\
0 \\
0 \\
0 \\
0 \\
0 \\
0 \\
0 \\
0 \\
0 \\
0 \\
\dot{0}\end{array}$ & 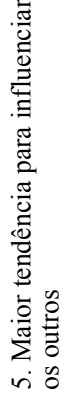 & 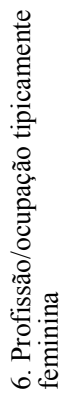 & 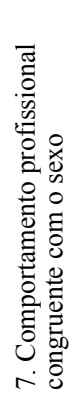 & 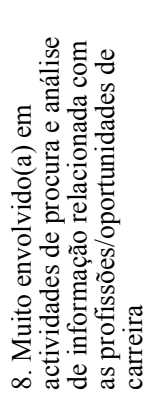 & 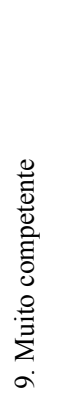 & 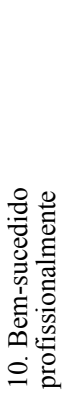 & 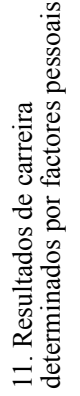 & 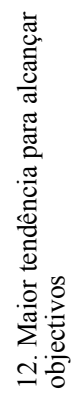 & 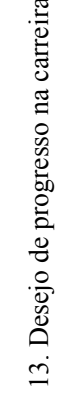 & 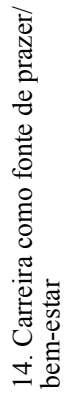 & 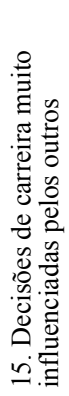 \\
\hline
\end{tabular}


O tratamento matemático da grelha de repertório, com base na análise de correspondências, permite obter uma variedade de medidas que expressam a associação entre os construtos, entre os elementos ou entre construtos e elementos. As medidas apresentadas neste estudo são: (a) Bieri; (b) Percentagem de variância explicada pelo primeiro factor (PVEPF); (c) Polarização; (d) Indefinição; (e) Ordenação ou Potência Discriminativa (PD); (f) Conflitos; (g) Dilemas Implicativos; (h) Número de Construtos Dilemáticos.

O índice de Bieri (Bieri, 1955) tem sido referido na literatura como um indicador da complexidade cognitiva que se baseia na diferenciação e multidimensionalidade da construção. É calculado pelo somatório de acordos de cotações da matriz, quando se comparam elemento a elemento e cada par de construtos, dividido pela pontuação máxima possível de acordo com o tamanho da grelha. Poucos acordos representam um menor índice Bieri, ou seja, valores mais próximos de zero do que de um e, consequentemente, um elevado grau de complexidade. De acordo com este índice, as pessoas cognitivamente mais complexas constroem o seu mundo a partir de maior diversidade nas dimensões de significado do que as que são cognitivamente menos complexas. O estudo deste índice nesta população revela que o índice de Bieri pode ser considerado baixo, indicando pouca relação entre os construtos, para valores inferiores a 0,24 , e elevado para valores superiores a 0,32 .

A PVEPF (Feixas, Lopez, Navarro, Tudela, \& Neimeyer, 1992; O'Keefe \& Sypher, 1981) é também considerada como um indicador de complexidade cognitiva ou diferenciação. Esta percentagem informa-nos sobre o poder explicativo do primeiro factor na construção de significados de carreira efectuada por uma dada pessoa. Sempre que o primeiro factor explica uma percentagem elevada da variância da construção cognitiva, considera-se que existe simplicidade ou unidimensionalidade na construção, ou seja, que o indivíduo atribui sentido às suas experiências e acontecimentos, sempre com base nas mesmas dimensões de significado pessoal. Se a explicação da variância é dividida por diferentes dimensões, então a construção pessoal é considerada como sendo mais diferenciada, ou seja, assume-se que a pessoa recorre a diferentes dimensões de significado para dar sentido a diferentes experiências e acontecimentos do seu mundo, neste caso, da sua carreira. Assim, uma elevada PVEPF reflecte indiferenciação, e uma baixa PVEPF reflecte diferenciação. $\mathrm{O}$ estudo deste índice nesta população revela que podemos considerar que o sistema de construção dos estudantes é muito diferenciado para valores inferiores a 42,61 , é moderadamente diferenciado, entre 42,61 e 57,76, sendo considerado indiferenciado a partir deste último valor.

O índice de Ordenação (Landfield \& Barr, 1976) mede o grau de ordenação de cada construto relativamente aos restantes. Calcula-se multiplicando o número de pontuações diferentes usadas para pontuar um construto ( 7 numa escala de 1 a 7) pela diferença entre a pontuação máxima e mínima atribuída a esse construto, dividindo pelo total de vezes que esse construto foi pontuado, ou seja, pelo número de elementos. O cálculo pode ser realizado em índices globais para todos os construtos, para todos os elementos e para construtos e elementos. Este índice foi definido pelo seu autor original como uma forma de medir a integração hierárquica do sistema de construção. $\mathrm{O}$ autor sugere que o grau de ordenação de cada construto indica o seu nível hierárquico no sistema. Contudo, Feixas (1988) considera que este índice é mais uma medida de flexibilidade do sistema de construção. Desta forma o mesmo autor chama-o de Potência Discriminativa, uma vez que ele indica a capacidade da pessoa para discriminar os vários elementos com base nos construtos que está a utilizar e vice-versa. Numa grelha com escala de 7 pontos, o índice de Potência Discriminativa total (média da PD de construtos e PD de elementos) pode assumir valores que variam entre 0 e 2,72. O estudo da Potência Discriminativa, nesta população, indica-nos que a construção dos sujeitos em torno da carreira pode ser considerada inflexível ou com menos poder discriminativo para valores inferiores a 1,24, e será flexível para valores superiores a 1,69 .

O índice de Polarização (Bonarius, 1977) é uma medida que indica a percentagem em que são atribuídas pontuações extremas no âmbito da pontuação de um construto (por exemplo, percentagem de 1 a 7 numa escala de 7 pontos). Tem sido entendido como uma medida de rigidez ou flexibilidade do sistema cognitivo. Quanto mais elevado for o índice, mais polarizada e rígida é a construção de significados. O estudo deste índice nesta população indica que podemos considerar que esta é polarizada para valores superiores a 41,67, e será mais flexível para valores inferiores a 18,23.

O índice de Indefinição (Feixas \& Cornejo, 1996) avalia a dificuldade do sujeito em situar os vários elementos em um ou outro polo dos construtos. Corresponde à percentagem de pontuações médias (" 4 " na escala de 7 pontos) atribuídas, na matriz dos dados. Uma percentagem elevada de indefinição indica que é difícil ao sujeito atribuir sentido de modo significativo aos elementos do âmbito de conveniência que está a construir, neste caso, no âmbito da carreira. Esta dificuldade pode significar ausência de conhecimento ou de envolvimento do sujeito, ou mesmo, confusão quanto ao modo como os outros significativos são. $\mathrm{O}$ estudo deste índice nesta população indica que pode ser considerado baixo para valores inferiores a 12,08, e será elevado para valores superiores a 28,75.

A medida de Conflitos (Slade \& Sheehan, 1979) informa sobre a inconsistência nas construções pessoais de significado. Esta medida calcula-se considerando tríades de construtos em relação às quais se avalia a compatibilidade ou incompatibilidade das relações, considerando-se uma correlação de 0,20. Existem duas possibilidades que podem criar conflito na construção: (a) quando numa tríade de construtos (A, B e C) todas as correlações entre os construtos (A/B, B/C e C/A) são negativas; (b) quando numa tríade de construtos (A, B e C) duas correlações são positivas (A/B e B/C), e uma é negativa $(A / C)$. Um exemplo do primeiro caso é o conflito obtido com uma estudante do ensino superior que 
apresenta uma correlação negativa entre ter a carreira como objectivo prioritário de vida e ser autónoma (A/B), uma correlação negativa entre ser autónoma e ter um comportamento profissional congruente com o sexo $(B / C)$ que, por sua vez, se correlaciona negativamente com o facto de ter a carreira como objectivo prioritário de vida (C/A). Digamos que estas três dimensões valorizadas pela estudante são incompatíveis de ocorrer em simultâneo, criando conflito. O segundo caso de conflitos pode ser exemplificado com o conflito de outro estudante que quanto mais se vê como dependente dos outros (A) mais valoriza a família como objectivo prioritário de vida (B), e quanto mais valoriza a família como objectivo prioritário de vida maior tendência tem para alcançar objectivos (C), contudo esta tendência para alcançar objectivos (C) não é compatível com a percepção de dependência dos outros (A). Estes conflitos nas relações entre construtos manifestam, assim, alguma incoerência na construção de significado das experiências pessoais.

Os Dilemas Implicativos (Hinkle, 1965) referem-se a um tipo de conflito cognitivo, resultante da construção da experiência pessoal com base em dimensões de significado (construtos) cuja relação se apresenta como incompatível e ameaçadora da identidade do sujeito (Feixas, Saúl, ÁvilaEspada, \& Sánchez Rodríguez, 2001). O conceito de Dilema Implicativo ajuda-nos a compreender o impasse que surge muitas vezes em situações de transição de vida, como a finalização da licenciatura, que implicam tomadas de decisão importantes e movimento de reconstrução pessoal. Entre os construtos que o estudante usa para construir a sua experiência, alguns deles, os construtos congruentes, definem a sua proximidade a um self ideal, enquanto os outros, os construtos discrepantes, definem a sua distância actual e o respectivo desejo de mudança em direcção a um self ideal. Os primeiros construtos são aqueles cujo polo escolhido para dar significado ao self estudante (actual), o mesmo que dá significado ao self ideal (por exemplo, determinado a despender tempo e energia com a carreira), enquanto os discrepantes são os construtos em que um dos polos define o self actual (por exemplo, carreira como objectivo prioritário de vida) e o outro polo define o self ideal (por exemplo, família como objectivo prioritário de vida). O Dilema Implicativo surge quando existe uma correlação positiva $(r>0,20)$ entre um construto discrepante e um construto congruente, ou seja, quando a mudança desejada pelo estudante, inerente ao construto discrepante (por exemplo, carreira como objectivo prioritário de vida/família como objectivo prioritário de vida), implica uma mudança não desejada com implicações na forma como se coloca face ao construto congruente.

Número de Construtos Dilemáticos refere-se ao número de construtos numa grelha em que o indivíduo pontua o self ideal com um ponto médio (4 numa escala de 1 a 7). Esta é uma medida que resulta da análise qualitativa da grelha de repertório. Estes construtos mostram que o sujeito teve dificuldade em definir como gostaria de ser em termos ideais, mostrando indefinição em relação àquela dimensão de significado.

\section{Procedimento}

Os procedimentos usados para a recolha de dados obedeceram às exigências e particularidades da investigação mais ampla em que este estudo se insere (Silva, 2008), pelo que passaremos a referir os aspectos centrais para este artigo. A selecção da amostra resultou de duas preocupações centrais: (a) obter uma amostra equitativa nas diversas características pessoais e do contexto de vida da população a estudar (estudantes universitários a frequentar, pela primeira vez e sem retenções, o último ano de licenciatura); (b) equilibrar a relação entre custos e benefícios nos procedimentos de recolha e seguimento da amostra, dada a natureza longitudinal do estudo. Por isso, foi seleccionada, como instituição-alvo para a realização do estudo a Universidade do Minho, dadas as facilidades antecipadas nesses procedimentos. Para a selecção dos participantes, utilizou-se o método aleatório, sendo que os participantes tinham que obedecer aos seguintes critérios: (a) frequentar o último ano de uma das licenciaturas da Universidade do Minho; (b) nunca ter repetido de ano no decorrer da licenciatura; (c) participar voluntariamente.

O plano de avaliação foi administrado em entrevistas individuais. Estas entrevistas iniciaram-se pela assinatura do consentimento informado, levantamento de dados pessoais, seguindo-se a entrevista da Grelha de Repertório da Carreira (Silva \& Taveira, 2005). O tempo estimado como necessário para a entrevista individual foi de 60 minutos para cada participante. No final da entrevista, a investigadora avaliou, ainda, a activação psicológica que a administração da grelha poderia provocar nos participantes, tentando prevenir algum mal-estar, ansiedade ou desconforto decorrente dessa activação. A avaliação ocorreu em dois momentos. $\mathrm{O}$ primeiro correspondeu ao início do ano escolar e o segundo, ao final do ano escolar. Os procedimentos seguidos para a segunda fase foram os mesmos da primeira fase. Todos os participantes que concluíram as duas fases do projecto de investigação receberam um certificado de colaboração no projecto de investigação.

\section{Análise de dados}

$\mathrm{Na}$ análise dos dados, procedeu-se ao cálculo dos índices da Grelha de Repertório de Carreira com o software RECORD 4.0 (Feixas \& Cornejo, 2002) construído com base na análise de correspondências. Todas as grelhas foram tratadas individualmente neste software informático, tendo os resultados obtidos, nos diferentes índices para cada sujeito, sido inseridos e analisados, no programa Statistic Program for Social Sciences (SPSS) versão 14.0 para Windows (SPSS, 2005). No estudo de medidas repetidas, também utilizamos os testes disponíveis no programa Statistic Program for Social Sciences (SPSS) versão 14.0 para Windows (SPSS, 2005). Concretamente, depois de estudar a distribuição das variáveis em estudo, optamos pelo T-teste de comparação de médias para amostras emparelhadas correspondentes aos dois momentos de avaliação do estudo: início e final do último ano da licenciatura. 


\section{Resultados}

A Tabela 1 apresenta os resultados do T-teste para amostras emparelhadas dos dois momentos de avaliação, para as medidas de estrutura: índice Bieri, Percentagem de variância explicada pelo primeiro factor (PVEPF), Potência Discriminativa (PD), Polarização e Indefinição.

Tabela 1

Bieri, PVEPF, Potência Discriminativa, Polarização e Indefinição nos dois momentos de avaliação $(n=80)$

\begin{tabular}{lccccc}
\hline & \multicolumn{5}{c}{ Momento de Avaliação } \\
\cline { 2 - 6 } & $\begin{array}{c}\text { Momento 1 } \\
(n=80)\end{array}$ & \multicolumn{2}{c}{$\begin{array}{c}\text { Momento } 2 \\
(n=80)\end{array}$} & $t$ \\
\hline \multicolumn{1}{c}{$p($ sig $)$} & $M$ & $D P$ & $M$ & $D P$ & $p$ \\
\hline Bieri & & & & & \\
$\quad 0,113$ & 0,29 & 0,082 & 0,30 & 0,071 & $-1,60$ \\
PVEPF & & & & & \\
$\quad 0,706$ & 50,32 & 13,373 & 49,70 & 13,372 & 0,38 \\
P. Discriminativa** & & & & & \\
$\quad 0,001$ & 1,47 & 0,890 & 1,32 & 0,346 & 3,62 \\
$\begin{array}{l}\text { Polarização } \\
\quad 0,131\end{array}$ & 32,02 & 16,372 & 29,93 & 15,526 & 1,53 \\
Indefinição** & & & & & \\
0,008 & 20,61 & 10,396 & 23,51 & 12,890 & $-2,72$ \\
\hline
\end{tabular}

${ }^{*} p<0,05{ }^{* *} p<0,01$

A leitura da Tabela 1 evidencia, nos dois momentos de avaliação do estudo, a existência de diferenciação moderada do sistema de construção de significados em torno da carreira $(42,60<\mathrm{PVEPF}<57,76)$. Além disso, a inter-relação entre esses construtos revela-se também moderada, nos dois momentos de avaliação, indicando uma capacidade relativa dos estudantes para associar as dimensões de significado no âmbito da carreira $(0,24<$ Bieri $<0,32)$. No que diz respeito às restantes medidas, no momento 1 , regista-se um valor médio de Polarização também moderado. Desta forma, os estudantes não parecem ter uma construção demasiado polarizada e rígida no domínio da carreira. No momento 2 , verifica-se uma ligeira redução da polarização, mas que não é estatisticamente significativa. Contudo, em termos de flexibilidade, medida pelo índice de Potência Discriminativa, verifica-se que, no momento 1 , os estudantes apresentam uma construção de significados em torno da carreira relativamente flexível. No entanto, no momento 2 , os estudantes diminuem de forma estatisticamente significativa o resultado no índice de Potência Discriminativa. Isto indica que, no final da licenciatura, os estudantes estão menos flexíveis e capazes de discriminar as pessoas significativas em termos de carreira com base nas dimensões de significado fornecidas. Por fim, o índice de Indefinição apresenta valores médios moderados $(12,1<$ Indefinição<28,75) nos dois momentos de estudo, revelando alguma indefinição do sistema de construção dos estudantes. Comparando os dois momentos de avaliação do estudo, verifica-se que os estudantes aumentam de forma estatisticamente significativa o índice de Indefinição, o que revela que, no final da licenciatura, os estudantes estão mais indecisos em relação às dimensões de carreira e em como as relacionar consigo e com outros significativos.

De uma forma geral, podemos considerar que os estudantes demonstram um perfil de organização cognitiva que tende para a indiferenciação e pouca associação face às dimensões de significado construídas no âmbito da carreira. Assim, o grupo de estudantes, na generalidade, dispõe, cognitivamente, de um número relativamente limitado de dimensões de significado face à carreira e demonstra alguma dificuldade em as relacionar de forma coerente. Isto pode gerar julgamentos mais rígidos, pouco definidos e coerentes no âmbito da carreira. Este perfil parece manter-se nos dois momentos de avaliação do estudo, revelando-se mais inflexível e indefinido no final da licenciatura.

Em seguida, apresenta-se a distribuição dos estudantes, nos dois momentos de avaliação do estudo, em termos de complexidade cognitiva medida pelo índice de Bieri e de flexibilidade medida pela Potência Discriminativa. Para tal, consideram-se três grupos (complexidade ou flexibilidade baixa, moderada e elevada) calculados com base nos pontos de referência obtidos nesta população.

A Tabela 2 apresenta esses resultados, em mulheres, homens e na amostra total.

\section{Tabela 2}

Distribuição dos estudantes por grupos de Complexidade Cognitiva e Flexibilidade, nos dois momentos de avaliação do estudo $(n=80)$

\begin{tabular}{|c|c|c|c|c|c|c|}
\hline & \multicolumn{3}{|c|}{ Momento 1} & \multicolumn{3}{|c|}{ Momento 2} \\
\hline & Mulheres $n(\%)$ & Homens $n(\%)$ & Total $n(\%)$ & Mulheres $n(\%)$ & Homens $n(\%)$ & Total $n(\%)$ \\
\hline & $n=49$ & $n=31$ & $n=80$ & $n=49$ & $n=31$ & $n=80$ \\
\hline \multicolumn{7}{|l|}{ Complexidade } \\
\hline Elevada & $12(24,5)$ & $11(35,5)$ & $23(28,8)$ & $6(12,2)$ & $9(29,0)$ & $15(18,8)$ \\
\hline Moderada & $25(51,0)$ & $10(32,3)$ & $35(43,8)$ & $26(53,1)$ & $15(48,4)$ & $41(51,3)$ \\
\hline Baixa & $12(24,5)$ & $10(32,3)$ & $22(27,5)$ & $17(34,7)$ & $7(22,6)$ & $24(30,0)$ \\
\hline \multicolumn{7}{|l|}{ Flexibilidade } \\
\hline Elevada & $14(28,6)$ & $9(29,0)$ & $23(28,8)$ & $6(12,2)$ & $7(22,6)$ & $13(16,3)$ \\
\hline Moderada & $21(56,8)$ & $16(43,2)$ & $37(46,3)$ & $20(40,8)$ & $15(48,4)$ & $35(43,8)$ \\
\hline Baixa & $14(28,6)$ & $6(19,4)$ & $20(25,0)$ & $23(46,9)$ & $9(29,0)$ & $32(40,0)$ \\
\hline
\end{tabular}


Como se pode observar pela leitura da Tabela 2, nos dois momentos, a elevada percentagem de estudantes com níveis moderados de complexidade cognitiva demonstra que os sistemas de construção dos estudantes não se demarcam nem em termos de diferenciação, nem em termos da associação entre as dimensões de significado. Isto pode sugerir um perfil de construção de alguma unidimensionalidade e paralelamente pouco relacionado. De realçar ainda, a este respeito, que, no final da licenciatura, um maior número de estudantes baixa a complexidade cognitiva. Assim, se a complexidade cognitiva prediz bem-estar e desenvolvimento saudável da identidade, alguns estudantes, no final da licenciatura, passaram a enfrentar mais dificuldades no desenvolvimento dessa identidade de carreira. Demonstram, ainda, maior dificuldade em organizar o sistema de construção de uma forma coerente, capazes de hierarquizar e discriminar as diferentes dimensões de carreira em relação aos elementos que estão a considerar.

A Tabela 3 apresenta os resultados do T-teste para amostras emparelhadas, para as medidas de conflito da estrutura do sistema de construção dos estudantes, nos dois momentos de avaliação do estudo.

Tabela 3

Número de conflitos, dilemas e construtos dilemáticos, nos dois momentos de avaliação $(n=80)$

\begin{tabular}{|c|c|c|c|c|c|c|c|c|}
\hline & \multicolumn{6}{|c|}{ Momento de Avaliação } & \multirow[b]{3}{*}{$t$} & \multirow[b]{3}{*}{$p($ sig $)$} \\
\hline & \multicolumn{3}{|c|}{$\underset{(n=80)}{\text { Momento } 1}$} & \multicolumn{3}{|c|}{$\underset{(n=80)}{\text { Momento } 2}$} & & \\
\hline & $M$ & $D P$ & Amp. & $M$ & $D P$ & Amp. & & \\
\hline Conflitos & 0,8 & 1,96 & {$[0,11]$} & 0,4 & 1,18 & {$[0,9]$} & 1,73 & 0,086 \\
\hline Dilemas** & 1,6 & 2,81 & {$[0,16]$} & 0,7 & 1,94 & {$[0,9]$} & 3,23 & 0,002 \\
\hline $\begin{array}{l}\text { Construtos } \\
\text { Dilem. }\end{array}$ & 2,9 & 1,93 & {$[0,8]$} & 3,2 & 2,45 & {$[0,14]$} & $-1,21$ & 0,229 \\
\hline
\end{tabular}

Como se pode observar pela leitura da Tabela 3, em relação às medidas de conflito de construção dos estudantes, verifica-se, entre os dois momentos de avaliação do estudo, uma redução da média do número de conflitos e de dilemas e um aumento da média do número de construtos dilemáticos. Em relação ao número de dilemas, essa redução é estatisticamente significativa. Esses resultados indicam que os estudantes, no final da licenciatura, apresentam menor inconsistência na construção de significado em termos de carreira, embora tenham maior dificuldade em se definirem do ponto de vista do ideal, relativamente às dimensões de carreira fornecidas.

\section{Discussão}

Este estudo teve como objectivo principal analisar mudanças na estrutura do self de carreira de estudantes do ensino superior, ao longo do último ano de licenciatura. De uma forma geral, em termos de estrutura cognitiva, os estudantes apresentam um sistema de construção de significados em torno da carreira moderadamente indiferenciado e polarizado. Estes resultados evidenciam uma moderada capacidade para operar e relacionar as diferentes dimensões de significado em torno de carreira. No final da licenciatura, contudo, e após a experiência de um estágio de seis meses em ambiente de trabalho profissional, os estudantes demonstram menor flexibilidade e maior indefinição na forma como se construíam a si mesmos e aos outros no âmbito da carreira. Isto sugere que, no final da licenciatura, os estudantes apresentam mais dificuldade em estabelecer uma estrutura hierárquica que discrimine e organize as diferentes dimensões da carreira de uma forma coerente. Isto pode revelar que esta fase da vida e do desenvolvimento em contexto académico implica novos desafios e a consideração de novas dimensões de significado que o sujeito não é capaz de integrar no seu self. A este respeito, Sanford (1962) chama a atenção para o papel das instituições de ensino superior, já que a presença ou ausência de duas condições básicas, o desafio e o apoio, juntamente com a personalidade dos estudantes, determinam a capacidade de resposta do aluno perante situações novas. Bondon e Bardagi (2008), num estudo recente acerca das necessidades dos estudantes universitários, concluíram que há um grande sentimento dos alunos pesquisados de que as graduações, regular e tecnológica, não preparam para o mundo do trabalho e a percepção de que o curso não é essencial para a atividade a ser desempenhada. Segundo as mesmas autoras, essas percepções, ainda que fruto de informações estereotipadas ou expectativas irrealistas quanto às profissões, devem servir como material de reflexão para as unidades de ensino, no sentido de que é necessário auxiliar o aluno a identificar as relações entre o que está sendo aprendido e as actividades a serem desenvolvidas no futuro.

A esse respeito, ainda, apesar de uma percentagem considerável de estudantes apresentar um perfil saudável de elevada complexidade cognitiva, no segundo momento de estudo, esta percentagem decresce e verifica-se uma redução da complexidade cognitiva e da flexibilidade ou capacidade discriminatória do sistema de construção. Estes aspectos revestem-se de especial importância porque nos permitem compreender as dificuldades que os indivíduos enfrentam, à saída do ensino superior, no planeamento de carreira e consequente integração no mercado de trabalho (Borralho, 2001; Lent, Hackett, \& Brown, 1996, 1999; Reitzle \& Vondracek, 2000). Ao mesmo tempo, a construção polarizada ou pouco flexível pode levar os estudantes a alternarem indiscriminadamente entre os poucos pontos de vista de carreira que possuem, demonstrando incoerência ou indecisão nos objectivos que formulam. Neste contexto, trabalhar psicologicamente os significados associados à carreira, promovendo a integração dos mesmos, num processo intencional de promoção de autoconhecimento, revela-se fundamental para que estes estudantes possam progredir para níveis de desenvolvimento de carreira mais avançados. A este respeito, King e Kitchener (1994), ao proporem o modelo do julgamento reflexivo que descreve a progressão desenvolvimental 
a partir da qual os indivíduos realizam e defendem assunções que lhes permitem compreender e aceitar a incerteza do real e do mundo do conhecimento, assumindo um determinado posicionamento, defendem que este desenvolvimento pode ser afectado de forma significativa pela qualidade do ambiente académico em que os estudantes estão inseridos. As autoras referidas sugerem medidas intencionais no contexto académico, envolvendo toda a comunidade, para promover o desenvolvimento deste pensamento reflexivo que implica níveis de complexidade cognitiva diferenciados e integrados.

A estrutura cognitiva dos estudantes pode estar a ser muito influenciada, também, pela instabilidade e até indefinição do mercado de trabalho e das carreiras em si, na actualidade. Além disso, torna-se aqui relevante considerar as actuais exigências que se impõem aos jovens em termos académicos e profissionais, nomeadamente, as decorrentes da implementação do sistema europeu de Bolonha (Declaração de Bolonha, 1999). Este sistema assenta na promoção da comparabilidade, da transparência e da legibilidade dos sistemas europeus de ensino superior. Para o efeito, as instituições de ensino superior tendem a centrar-se na reorganização dos graus e diplomas, na implementação dos instrumentos que promovam a mobilidade e a empregabilidade (sistema de créditos ECTS, contrato de estudos, escala europeia de comparabilidade de classificações e suplemento ao diploma), bem como no desenvolvimento de mecanismos que garantam a qualidade e acreditação dos seus cursos. Todo este processo de mudança e reestruturação pode implicar que os estudantes passem a considerar todo um conjunto de novas dimensões, inclusive de valores de carreira para os quais ainda não estão preparados. Desta forma, a estrutura cognitiva dos sujeitos protege-se na rigidez (onde preservam as dimensões que são muito relevantes para eles), indefinição e indiferenciação.

Focando-nos, agora, nas medidas de conflito de construção dos estudantes, os resultados indicam que os estudantes, no final da licenciatura, apresentam menor inconsistência na construção de significado em termos de carreira, embora tenham maior dificuldade em se definirem do ponto de vista do ideal, relativamente às dimensões de carreira fornecidas. Com efeito, a estrutura de conflito parece ser substituída pela indefinição que deixa em aberto a possibilidade de cedência em relação a determinadas dimensões de significado que, no início do ano lectivo, eram muito importantes, embora promotoras de colisão com outras dimensões de significado em torno da carreira, em termos ideais, para os estudantes. $\mathrm{O}$ contacto com o mercado de trabalho proporcionado pelo estágio e a interpretação que os estudantes fizeram dessa experiência podem ter contribuído para aumentar esta indefinição/indecisão em relação às dimensões que os estudantes aspiram alcançar. Harter $(1999,2003)$ refere, a respeito dos conflitos, que há factores contextuais, e não só cognitivodesenvolvimentistas, a influenciar a própria organização e estrutura dos atributos percebidos. Neste caso, então, o contacto com o contexto pode ter aumentado o realismo dos estudantes a respeito do mesmo, ajudando-os a resolver alguns dos conflitos no confronto com os mesmos, ainda que, para tal, o sujeito tenha de se manter num estado de indefinição.

\section{Conclusão}

Os resultados deste estudo sugerem que, no final da licenciatura, os estudantes têm mais dificuldade em construir significados de carreira do que no início do ano escolar. Dado que, nesse período de tempo, os estudantes vivenciaram uma experiência de estágio, num contexto de trabalho, podemos depreender que essa experiência pode ter um impacto decisivo nas dimensões que contribuem para a forma como constroem as suas carreiras, à saída do ensino superior. É de salientar, também, que parece fundamental que a Universidade, para além da dimensão académica e técnica, proporcione aos alunos momentos de reflexão e de integração dessas novas experiências, de uma forma protegida, que lhes permitam caminhar para situações de maior autonomia. Neste contexto, parecenos relevante, também, o esforço que os estudantes finalistas realizam para se adaptarem às novas exigências de uma fase de transição para um novo ciclo de vida, como o demonstram, por exemplo, os resultados obtidos nos índices de conflito do $\mathrm{Eu}$ de carreira. Concretamente, no final da licenciatura, os estudantes deste estudo parecem resolver alguns dos seus dilemas e conflitos de carreira, mantendo-se mais indecisos quanto às suas aspirações. Seria importante analisar com os mesmos outras formas alternativas e mais positivas de gerir a sua carreira, ao longo da permanência no ensino superior.

\section{Referências}

Bardagi, M. P. (2007). Evasão e comportamento vocacional de universitários: Estudos sobre o desenvolvimento de carreira na graduação. Tese de doutorado nãopublicada, Universidade Federal do Rio Grande do Sul, Porto Alegre.

Bieri, J. (1955). Cognitive complexity-simplicity and predictive behavior. Journal of Abnormal and Social Psychology, 51, 263-286.

Bonarius, H. (1977). The interaction model of communication: Through experimental research towards existential relevance. In A. W. Landfield \& J. K. Cole (Eds.), Nebraska symposium on motivation 1976: Vol. 26. Personal construct psychology. Lincoln, NE: University of Nebraska Press.

Bondan, A. P., \& Bardagi, M. P. (2008). Comprometimento profissional e estressores percebidos por graduandos regulares e tecnológicos. Paideia (Ribeirão Preto), 18, 581-590.

Borralho, A. M. (2001). Didáctica da matemática e formação inicial: Um estudo com três futuros professores. Tese de doutorado não-publicada, Universidade de Évora, Évora, Portugal. 
Brott, P. E. (2001). The storied approach: A postmodern perspective for career counseling. Career Development Quarterly, 49, 304-313.

Declaração de Bolonha. (1999). Joint declaration of the european ministers of education convened in Bologna on the 19th of June. Recuperado em 15 janeiro 2005, de http://europa.eu.int/comm/education/policies/educ/ bologna/bologna_en.html

Decreto-Lei Portaria ${ }^{\circ} 256$. (2005, 16 de Março). Define a classificação nacional de áreas de educação e formação. Lisboa: Diário da República Portuguesa.

Feixas, G. V. (1988). L'an 'lisi de construccions personals en textos de significacis psicológica (Tese de doutorado microfichada, n. 328). Barcelona, Espanha: Publicacions Universitat de Barcelona.

Feixas, G., \& Cornejo, J. M. (1996). Manual de la técnica de Rejilla: Mediante el Programa RECORD v. 2.0 (2a ed.). Barcelona, Espanha: Paidós.

Feixas, G., \& Cornejo, J. M. (2002). RECORD v. 4.0: Análisis de correspondencias de constructos personales. Barcelona, Espanha: Psimedia.

Feixas, G., Lopez, J., Navarro, J., Tudela, M., \& Neimeyer, R.A. (1992). A study of reliability among grid measures. International Journal of Personal Construct Psychology, 5, 353-367.

Feixas, G., Saúl, L. A., Ávila-Espada, A., \& Sánchez Rodríguez, V. (2001). Implicaciones terapéuticas de los conflictos cognitivos. Revista Argentina de Psicología Clínica, 10(1), 5-13.

Fernandes, E. (2001). Grelha de repertório. In E. Fernandes \& L. Almeida (Orgs.), Métodos e técnicas de avaliação: Contributos para a prática e investigação psicológicas (pp. 77- 108). Minho, Portugal: Universidade do Minho, Centro de estudos em Educação e Psicologia.

Fernandes, E., \& Gonçalves, O. (1997). Exploração de construtos pessoais sobre o self em mudança: Um estudo preliminar com sujeitos depressivos e agorafóbicos. Psicologia: Teoria, Investigação e Prática, 2(1), 33-52.

Fernandes, E., Senra, J., \& Feixas, G. (2009). Psicoterapia Construtivista: Um modelo centrado em dilemas. Braga, Portugal: Psiquilíbrios Edições.

Harter, S. (1985). Competence as a dimension of selfevaluation: Toward a comprehensive model of selfworth. In R. Leahy (Ed.), The development of the self ( $\mathrm{pp}$. 55-121). New York: Academic Press.

Harter, S. (1988). The construction and conservation of the self: James and Cooley revisited. In D. Lapsley \& F. Power (Eds.), Self ego and identity: Integrative approaches (pp. 43-70). New York: Springer Verlag.

Harter, S. (1999). The construction of the self: Developmental perspective. New York: The Guilford Press.

Harter, S. (2003). The development of self representations during childhood and adolescence. In M. Leary \& J. Tangney (Eds.), Handbook of self and identity (pp. 610-642). New York: The Guilford Press.
Hinkle, D. N. (1965). The change of personal constructs from a view-point of a theory of construct. Tese de doutorado não-publicada, The Ohio State University, Ohio.

Kelly, G. A. (1955). The psychology of personal constructs: Vols. 1-2. New York: W. W. Norton.

King, P. M., \& Kitchener, K. S. (1994). Developing reflective judgment. San Francisco: Jossey-Bass Publishers.

Kohlberg, L. A. (1990). Which postformal levels are stages? In M. Commons, C. Armon, L. Kolhberg, F. Richards, T. Grozer, \& J. Sinnott (Eds.), Adult development: Vol. 2. Models and methods in the study of adolescent and adult thought. New York: Praeger.

Landfield, A. R., \& Barr, M. A. (1976). Ordination: A new measure of concept organization. Licoln, OR: University of Nebraska Press.

Leitão, L. M, Paixão, M. P, Silva, J. T., \& Miguel, J. P. (2000). Apoio psicossocial a estudantes do ensino superior: Do modelo teórico aos níveis de intervenção. Psicologia, 14(2), 123-147.

Lent, R. W., Brown, S. D., \& Hackett, G. (2002). Social cognitive career theory. In D. Brown (Ed.), Career choice and development (4th ed., pp. 255-311). San Francisco: Jossey-Bass.

Lent, R. W., Hackett, G., \& Brown, S. D. (1996). A social cognitive framework for studying career choice and transition to work. Journal of Vocational Education Research, 21(4), 3-31.

Lent, R. W., Hackett, G., \& Brown, S. D. (1999). A social cognitive view of school to work transition. The Career Development Quarterly, 47, 297-311.

Marcia, J. E. (1966). Development and validation of egoidentity status. Journal of Personality and Social Psychology, 3, 551-559.

Marcia, J. E. (1976). Identity six years after: A follow-up study. Journal of Youth and Adolescence, 5, 145-160.

Marcia, J. E. (1980). Identity in adolescence. In J. Adelson (Ed.), Handbook of adolescence psychology. New York: Wiley.

O’Keefe, D. J., \& Sypher, H. E. (1981). Cognitive complexity measures and the relationship of cognitive complexity to communication. Human Communication Research, 8, 72-92.

Reitzle, M., \& Vondracek, F. W. (2000). Methodological avenues for the study of career pathways. Journal of Vocational Behavior, 57, 445-467.

Sanford, N. (1962). The American college: A psychological and social interpretation of the higher learning. New York: John Wiley \& Sons.

Savickas, M. L. (2004). Um modelo para avaliação de carreira. In L. Leitão (Org.), Avaliação psicológica em orientação escolar e profissional (pp. 21-44). Coimbra, Portugal: Quarteto.

Silva, A. D. (2008). A construção de carreira no ensino superior. Tese de doutorado não- publicada, Universidade do Minho, Braga, Portugal. 
Silva, A. D., \& Taveira, A. D. (2005). Grelha de repertório de carreira $(G R C)$. Instrumento de avaliação não-publicado, Universidade do Minho, Braga, Portugal.

Slade, P. D., \& Sheehan, M. J. (1979). The measurement of conflict in repertory grids. British Journal of Psychology, 70, 519-524.

SPSS developer's guide. (2005) [Computer software]. Chicago, IL: SPSS Inc.

Winter, D. A. (2003). Repertory grid technique as a psychotherapy research measure. Psychotherapy Research, 13, 25-42.

Ana Daniela Silva é Doutorada em Psicologia Vocacional pela Escola de Psicologia da Universidade do Minho, bolsista da Fundação para a Ciência e Tecnologia.

Maria do Céu Taveira é Professora Auxiliar da Escola de Psicologia da Universidade do Minho, campus Gualtar.

Eugénia Ribeiro é Professora Auxiliar da Escola de Psicologia da Universidade do Minho, campus Gualtar.

Recebido: 13/01/2009

$1^{a}$ revisão: 12/05/2009

$2^{a}$ revisão: 05/07/2009

Aceite final: 14/08/2009 